\title{
Endodontic Management of a Maxillary Lateral Incisor fused with Supernumerary Tooth using Spiral Computed Tomography as Diagnostic Aid
}

\author{
${ }^{1}$ Deepti Dua, ${ }^{2}$ Ankur Dua, ${ }^{3}$ Anand C Patil
}

\begin{abstract}
Fusion is a developmental anomaly in tooth morphology, which may be due to either union of two separate tooth buds or partial splitting of a single tooth bud. A thorough understanding of root canal anatomy is an essential prerequisite for a successful endodontic treatment. This case report describes successful endodontic management of a maxillary left lateral incisor fused with a supernumerary tooth using spiral computed tomography (SCT) as a diagnostic aid. A patient reported with a painless swelling in the maxillary anterior region associated with a fused maxillary lateral incisor. Spiral CT was performed to better understand the complex root canal anatomy. Endodontic treatment was done using methyl trioxide aggregate (MTA) as apical plug and obturation by thermoplasticized gutta-percha. The tooth was completely asymptomatic at 1-year follow-up showing signs of healing. The present case report emphasizes on the importance of three-dimensional (3D) imaging techniques, such as SCT in cases of developmental anomalies having complicated root canal morphology for successful endodontic therapy.
\end{abstract}

Keywords: Fusion, Mineral trioxide aggregate, Spiral computed tomography, Supernumerary tooth.

How to cite this article: Dua D, Dua A, Patil AC. Endodontic Management of a Maxillary Lateral Incisor fused with Supernumerary Tooth using Spiral Computed Tomography as Diagnostic Aid. World J Dent 2016;7(4):199-202.

Source of Support: Nil

Conflict of Interest: None

\section{INTRODUCTION}

Development of an ideal dentition depends on many factors. Disorders of development of teeth can be classified according to the abnormalities in shape, such as dens invaginatus, talon cusp, dens evaginatus, gemination, fusion, or abnormalities in number, such as supernumerary teeth. Double tooth presents as one of the

\footnotetext{
1,2 Lecturer, ${ }^{3}$ Professor and Head

1,2Department of Conservative Dentistry, Ibn Sina National College for Medical Studies, Jeddah, Kingdom of Saudi Arabia

${ }^{3}$ Department of Conservative Dentistry and Endodontics KLE Viswanath Katti Institute of Dental Sciences, Belgaum Karnataka, India

Corresponding Author: Ankur Dua, Lecturer, Department of Conservative Dentistry, Ibn Sina National College for Medical Studies, Jeddah, Kingdom of Saudi Arabia, Phone: +919891188682, +966598566552, e-mail: ankurdua@gmail.com
}

rare anomalies affecting the shape of the tooth, which denotes embryological conditions like gemination and fusion. Although the cause is unknown, but trauma has been suggested as a possible cause; genetic factors may also be involved. ${ }^{1}$ The incidence of double tooth ranges from 0.14 to $5.0 \%$ in worldwide population, with no sex predilection. Unilateral presentation is more common than the bilateral. ${ }^{2}$ Pindborg defined fusion as the union between dentin and/or enamel of two or more separate developing teeth. ${ }^{3}$ Depending on the stage at which the tooth germs join together, the fusion may be complete or incomplete. If the contact occurs early, at least before calcification begins, complete fusion occurs, forming a single large tooth. If the tooth germs come into contact when a portion of tooth crown has completed its formation, then incomplete fusion occurs. Such teeth may have either two pulp chambers with their respective root canals or a single root canal system. If fusion occurs between a normal and a supernumerary tooth, it becomes difficult to differentiate it from gemination. ${ }^{1}$ Pindborg $^{3}$ described gemination as the malformation of a single tooth bud, resulting in an anomalous tooth within the normal complement of teeth. Gemination occurs when a single tooth germ attempts to divide, resulting in a large single tooth with a bifid crown and a common root canal.

Endodontic treatment of double teeth has been a constant challenge for the practitioners because of variations in root canal anatomy. Intraoral periapical (IOPA) radiographs provide only the two-dimensional (2D) image of root canal which may not be sufficient enough for understanding the complex morphology of pulp canal space in anomalous dentition, such as fused teeth. Newer diagnostic techniques, such as spiral computed tomography (SCT) or volume acquisition $\mathrm{CT}$, which provide three-dimensional (3D) images, should be used in such cases for better evaluation of the root canal anatomy and hence a successful endodontic therapy.

The present case report describes successful endodontic management of a maxillary left lateral incisor fused with a supernumerary tooth with the aid of SCT.

\section{CASE REPORT}

A 28-year-old female patient reported to the Department of Conservative Dentistry and Endodontics with the 
complaint of painless swelling in left maxillary anterior region since 1 year. The medical and family histories were noncontributory. All vital signs were within normal limits. Clinical examination revealed a diffuse palatal swelling extending from the distal aspect of left central incisor to the distal of first premolar; the swelling was fluctuant on palpation. Hard tissue examination revealed a temporary restoration on palatal surface of maxillary left central incisor and abnormal crown morphology of maxillary left lateral incisor (Fig. 1A). There was an increased mesiodistal diameter with a supernumerary tooth attached palatal and mesial to the maxillary left lateral incisor (Fig. 1B). The labial and palatal surfaces of both lateral incisor and the supernumerary tooth were found to be caries free. No discomfort was present on vertical and horizontal percussion or palpation of tooth. The tooth exhibited no mobility. Thermal and electric pulp testing showed negative response in relation to the left central incisor, left lateral incisor, and the supernumerary tooth.

The preoperative periapical radiograph revealed a well-defined periapical radiolucency approximately $4 \mathrm{~cm}$ in diameter involving the lateral incisor and the supernumerary tooth which were fused along the entire root length (Fig. 1C). The pulp canal space exhibited a complex structure but the exact anatomy could not be understood from a 2D radiograph. All IOPA radiographs were depicting a radiopaque foreign body which was the permanent jewelry worn by the patient in the nose. Hence, care was taken to avoid its interference over the area of concern.

However, for better understanding of the root canal anatomy, a 3D dental imaging was planned for the tooth. Informed consent was obtained from the patient and a multislice SCT of maxilla was performed using Dentascan software (GE HealthCare, Milwaukee, WI, USA). The involved tooth was focused and the morphology was obtained in axial, transverse, and sagittal sections of $0.5 \mathrm{~mm}$ thickness along with 3D reconstructed images. Images revealed that the lateral incisor and the supernumerary tooth had two separate pulp chambers, but the pulp canal space shifted majorly into the supernumerary tooth as it progressed from coronal to apical section of the roots to join into a common large apical foramen in the supernumerary tooth corresponding to Weine's type II configuration (Figs 2A to D).

The treatment plan included root canal therapy with respect to the central incisor, lateral incisor, and the supernumerary tooth. Surgical management of the cyst was planned which included enucleation.

Local anesthesia was administered and rubber dam was applied. Endodontic access cavity was made on the
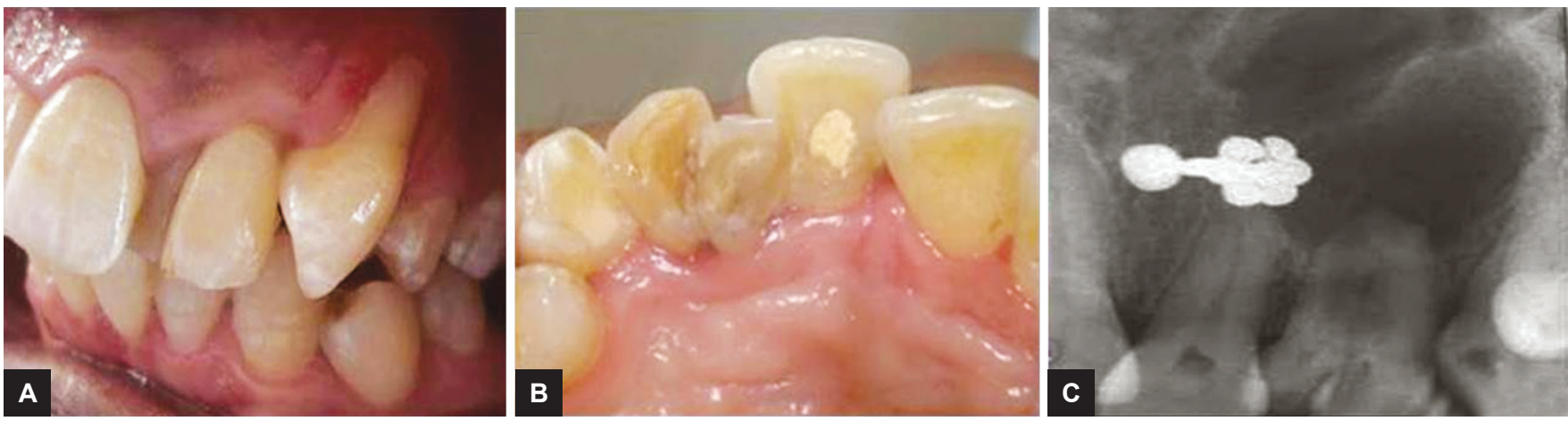

Figs $1 \mathrm{~A}$ to $\mathrm{C}$ : Preoperative photographs: $(\mathrm{A})$ Labial view; $(\mathrm{B})$ palatal view; and $(\mathrm{C})$ intraoral periapical radiograph showing irregular morphology and a large periapical radiolucency in relation to tooth nos. 21, 22
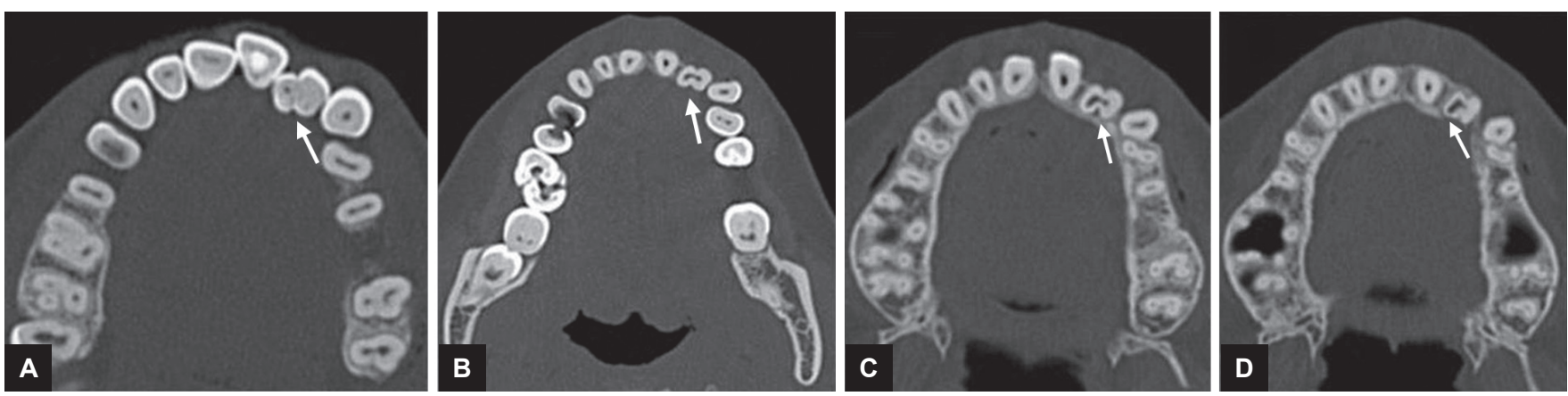

Figs 2A to D: Spiral CT images of maxilla: (A) Axial view of the coronal third section of the crown of the fused left lateral incisor and the supernumerary tooth; (B) axial view of the cervical third section of the roots of the fused left lateral incisor and the supernumerary tooth, (C) axial view of the middle third section of the roots of the fused left lateral incisor and the supernumerary tooth; and (D) axial view of the apical third section of the roots of the fused left lateral incisor and the supernumerary tooth 
palatal surfaces of the central incisor, lateral incisor, and supernumerary tooth using Endo Access bur no. A0164 (Dentsply Maillefer, Ballaigues, Switzerland). Following pulp extirpation using barbed broaches (Dentsply Maillefer, Ballaigues, Switzerland) and K-files (Kendo, VDW, Germany), coronal flaring was done with ProTaper SX (Dentsply Maillefer, Ballaigues, Switzerland). Working length was determined with apex locator (Root ZX, J Morita) and confirmed with radiographs. Cleaning and shaping was done using ProTaper rotary files (Dentsply Maillefer, Ballaigues, Switzerland) till F5, ISO size 50 along with circumferential filing. For root canal irrigation, $3 \mathrm{~mL}$ of $3 \%$ sodium hypochlorite (Vishal Dentocare, Ahmedabad, India) was used in between instruments. Canal was thoroughly dried with paper points and filled with calcium hydroxide medicament, Metapex (Meta Biomed Co., Korea) for 15 days.

Following 15 days, medicament was removed; canal was checked for any exudates in the central incisor and the lateral incisor. Canal was dried and gutta-percha obturation was completed in the central incisor whereas an apical plug of $4 \mathrm{~mm}$ was placed in the root canal of fused lateral incisor using mineral trioxide aggregate (ProRoot MTA, Dentsply Maillefer, Ballaigues, Switzerland) and confirmed with an IOPA radiograph (Fig. 3A). Coronal access was sealed with a sterile cotton pellet and a temporary cement (Cavit, 3M ESPE Dental AG, Seefeld/Oberbay, Germany). After 24 hours, setting of MTA was confirmed and obturation was completed with thermoplasticized injection fill gutta-percha (Elements Obturation Unit, SybronEndo, Orange, CA, USA) and AH Plus sealer (Dentsply DeTrey, Weybridge, UK). The access cavity was sealed with composite resin (Filtek Z350, 3M ESPE, St. Paul, MN, USA) in both central and lateral incisors.

The patient was not keen for the esthetic management of the anomaly, so only endodontic therapy was performed. The tooth was completely asymptomatic at 1 year recall visit showing signs of healing (Fig. 3B).
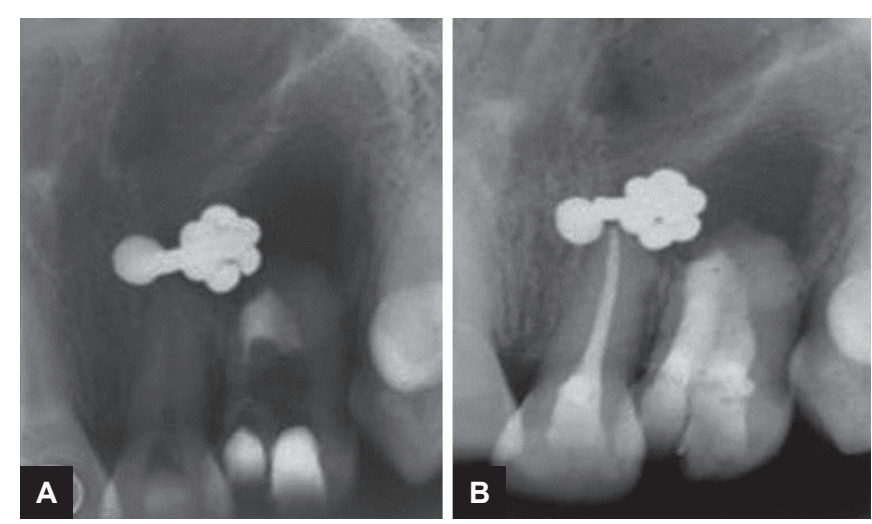

Figs 3 A and $B$ : (A) Intraoral periapical radiograph showing MTA apical plug in tooth no. 22; and (B) follow-up IOPA radiograph at 1 year recall

\section{DISCUSSION}

Management of a malformed tooth, such as fusion is often a challenge to the dentist. Fusion is often confused with the process of gemination, especially when a supernumerary tooth fuses with a permanent tooth. These two can be differentiated by the following parameters ${ }^{4}$ :

- Morphology: Gemination results in mirror images of the coronal halves, whereas fusion takes place at an angle.

- Anatomy: Fused teeth would mostly have separate pulp chamber and root canals while geminated teeth usually have one large pulp canal.

- Location: Fusion is common in mandible but fusion between a supernumerary and a normal tooth is more common in maxilla. Gemination is, however, more commonly seen in maxilla.

- Number of teeth: According to Mader, ${ }^{5}$ the "two tooth rule" may be helpful in differentiating fusion from gemination. If fused teeth are counted as one and the number of teeth in the dental arch is less, then it may be considered as fusion. However, when the anomalous tooth is counted as one and the number of teeth in dental arch is normal, then it can be either gemination or fusion between normal and a supernumerary tooth. In the present case, according to the "two tooth rule" and the SCT findings, diagnosis of fusion between maxillary lateral incisor and a supernumerary tooth was made. Fused teeth are generally asymptomatic and sometimes patient might retain the anomalous structure also without any problem. However, such double teeth may not only compromise the function and esthetics but can also be associated with various problems, such as carious lesions along the line of fusion or periodontal-endodontic involvement. Endodontic treatment in such teeth presents a great challenge to the dentist due to unpredictable root canal anatomy.

Radiographs are an indispensable diagnostic aid in endodontics. However, in such cases, conventional radiographs have limitations primarily because of the inherent projection of a 3D anatomy into a 2D image, which leads to superimposition, geometric distortion of the image, and hence misinterpretation and/or misdiagnosis. ${ }^{6,7}$ Tachibana and Matsumoto ${ }^{8}$ found that CT allowed multidirectional observation of the morphology of roots, root canals, and the tooth. The newer 3D diagnostic technology of SCT allows to obtain a large volume of data in seconds and offers more rapid examination time with the effective dose in the range of 1 to $30 \mathrm{mSv}$, which is much less than conventional CT. ${ }^{9}$ In the present case, guidelines proposed by Diederichs et $\mathrm{al}^{10}$ were used that decreased the radiation dosage to $0.56 \pm 0.06 \mathrm{mGy}$, which is equivalent to a single panoramic radiograph. 
The SCT simultaneously involves the patient translation through the $X$-ray source and the continuous rotation of the source detector assembly. Thus, the raw projection data acquired by the SCT can be viewed as multiplanar or 3D reconstructions. ${ }^{11}$ It also allows for reconstruction of overlapping structures which, thereby, enhances resolution of small objects.

Mineral trioxide aggregate apical plug of $4 \mathrm{~mm}$ was placed in the fused lateral incisor since it has shown to have a superior sealing ability, good marginal adaptation, high degree of biocompatibility, and a reasonable setting time. ${ }^{12}$ Moreover, MTA can be used in cases where there is exudate since it can set in the presence of moisture in the root canal. This property is important in teeth with necrotic pulps and periapical lesions. However, in the present case, calcium hydroxide intracanal medicament was placed to limit bacterial contamination. The middle and cervical part of canals were obturated with injection fill thermoplasticized gutta-percha and a resin sealer to ensure a 3D obturation.

In the present case, the SCT allowed for complete understanding of the root canal morphology. This enabled us to perform a successful cleaning and shaping and obturation of the complex root canal space. The SCT has been successfully used in clinical dentistry for confirmatory diagnosis of morphoanatomic aberrations in root canals. ${ }^{13}$ In recent years, cone beam CT has emerged as an innovation in the field of dentistry imaging, allowing for various 3D reconstructions. ${ }^{14}$ The new generation equipments permit the visualization of soft and hard tissues, surpassing conventional images in relation to the linear measurements of maxillaries, location and extension of dental resorptions, radicular position, presence of radicular fractures, and diagnosis of bone lesions.

\section{CONCLUSION}

Endodontic management of fused teeth may pose a constant challenge for the practitioners because of variations in root canal anatomy. Newer diagnostic techniques, such as SCT which provide 3D images should be used in such cases for better evaluation of the root canal anatomy and hence a successful endodontic therapy.

\section{REFERENCES}

1. Rajendran R, Sivapathasundharam B. Developmental disturbances of oral and paraoral structures: disturbances of development and growth. In: Shafer WG, Hine MK, Levy BM, editors. Shafer's text book of oral pathology. 5th ed. Amsterdam: Elsevier; 2006. p. 3-112.

2. Brook AH, Winter GB. Double teeth. A retrospective study of "geminated" and "fused" teeth in children. Br Dent J 1970 Aug;129(3):123-130.

3. Pindborg, JJ. Pathology of the dental hard tissues. Philadelphia: W.B. Saunders; 1970. Chapter 1, Abnormalities of tooth morphology; p. 48-57.

4. Schuurs AH, van Loveren C. Double teeth: review of the literature. ASDC J Dent Child 2000 Sep-Oct;67(5):313-325.

5. Mader CL. Fusion of teeth. J Am Dent Assoc 1979 Jan;98(1): 62-64.

6. Bender IB, Seltzer S. Roentgenographic and direct observation of experimental lesions in bone: I. 1961. J Endod 2003 Nov;29(11):702-706.

7. Bender IB, Seltzer S. Roentgenographic and direct observation of experimental lesions in bone: II. 1961. J Endod 2003 Nov;29(11):707-712.

8. Tachibana H, Matsumoto K. Applicability of X-ray computerized tomography in endodontics. Endod Dent Traumatol 1990 Feb;6(1):16-20.

9. Zoetelief J, Geleijns J. Patient doses in Spiral CT. Br J Radiol 1998 Jun;71(846):584-586.

10. Diederichs CG, Engelke WG, Richter B, Hermann KP, Oestmann JW. Must radiation dose for CT of the maxilla and mandible be higher than that for conventional panoramic radiography? AJNR Am J Neuroradiol 1996 Oct;17(9): 1758-1760.

11. Kalender WA, Seissler W, Klotz E, Vock P. Spiral volumetric CT with single-breath-hold technique, continuous transport, and continuous scanner rotation. Radiology 1990 Jul;176(1):181-183.

12. Torabinejad M, Smith PW, Kettering JD, Pitt Ford TR. Comparative investigation of marginal adaptation of mineral trioxide aggregate and other commonly used root-end filling materials. J Endod 1995 Jun;21(6):295-299.

13. Rani AK, Metgud S, Yakub SS, Pai U, Toshniwal NG, Bawaskar N. Endodontic and esthetic management of maxillary lateral incisor fused to a supernumerary tooth associated with a talon cusp by using spiral computed tomography as a diagnostic aid: a case report. J Endod 2010 Feb;36(2):345-349.

14. Ball RL, Barbizam JV, Cohenca N. Intraoperative endodontic applications of cone-beam computed tomography. J Endod 2013 Apr;39(4):548-557. 